\title{
Fatores Internos como Determinantes da Competitividade no Comércio Internacional: um Enfoque Gravitacional
}

\section{Internal Factors as Determinants of Competitiveness in International Trade: a Gravity Approach}

\author{
Josiane Souza de Paula** \\ Orlando Monteiro da Silva***
}

\begin{abstract}
Resumo: A consideração de fatores internos, além das variáveis de fronteira, tem relevância crescente nos estudos acerca do comércio internacional. Diante disso, propõe-se neste estudo uma definição ampliada do conceito de facilitação de comércio que permite avaliar a contribuição de indicadores de competitividade internos das nações sobre os fluxos de comércio internacional. Assim, os objetivos deste estudo são identificar e analisar os efeitos de indicadores de competitividade relativa dos países sobre os fluxos comerciais bilaterais. Os indicadores considerados são: desempenho econômico, eficiência dos governos, eficiência dos negócios e infraestrutura das diferentes nações. Estimam-se equações de gravidade com dados de comércio de 59 países, no período de 1997 a 2011 . De maneira geral, os resultados são robustos; em particular, demonstram que o nível de eficiência nos negócios indica maior impacto no comércio, em comparação com os demais indicadores.
\end{abstract}

Palavras-chave: Comércio internacional. Indicadores de competitividade. Modelo gravitacional.

\begin{abstract}
The consideration of internal factors, besides the variables of the border has increasing relevance in studies of international trade. Therefore, we propose an expanded definition of trade facilitation, which allows to evaluate the contribution of competitiveness indicators internal of nations on international trade flows. The aim of this study was to identify and analyze the effects of indicators of relative competitiveness of countries on bilateral trade flows. The indicators were considered: economic performance, government efficiency, business efficiency and infrastructure of different nations. Gravity equations with trade data of 59 countries in the period 1997 to 2011 were adjusted. Overall, the results were robust and, in particular, demonstrated that the level of business efficiency indicates a greater impact on trade compared to other indicators.
\end{abstract}

Keywords: International trade. Competitiveness indicators. Gravitational models.

JEL Classification: F13; F19; C23.

\footnotetext{
** Doutoranda em Economia pela Universidade Federal de Uberlândia (UFU). E-mail: josiane_udi@ yahoo.com.br

*** Professor do Departamento de Economia da Universidade Federal de Viçosa (UFV). E-mail: odasilva@ufv.br
} 


\section{1 lntrodução}

Em um período de intensificação do comércio internacional e da liberalização comercial, a busca pela redução das barreiras (tarifárias e não tarifárias) ao comércio tem sido tema constante de discussão entre diferentes países. Assim, dadas as frequentes negociações e acordos multilaterais de comércio sob o compromisso da Organização Mundial de Comércio $(\mathrm{OMC})$, que levaram à redução dessas barreiras, houve aumento da importância relativa de procedimentos de fronteira ${ }^{1}$ como geradores dos custos de comercialização ligados ao comércio internacional. Foi nesse ambiente que a facilitação de comércio passou a ter relevância no cenário político internacional.

A discussão sobre a facilitação de comércio ganhou relevância na $\mathrm{OMC}$ a partir da Conferência de Singapura (1996), como estudo exploratório e analítico acerca da simplificação dos procedimentos comerciais (WORLD TRADE ORGANIZATION, 2011), mas somente em 2004 os países membros concordaram em iniciar as negociações (DUVAL, 2008). Contudo, além dos facilitadores de comércio, que são basicamente variáveis de fronteira, ${ }^{2}$ há de se atentar para variáveis internas ${ }^{3}$ que têm sido cada vez mais chamadas a auxiliar nas decisões comerciais internacionais.

No debate acadêmico, a direção de estudos teóricos e empíricos relacionados com a facilitação de comércio tem ganhado espaço dentro da teoria econômica do comércio internacional (ALBURO, 2008; DUVAL; UTOKTHAM, 2009, 2010; SHEPHERD; WILSON, 2008; SOUZA, 2009). Duval (2008) mostra que um dos princípios fundamentais de facilitação de comércio é a transparência. Ao implementar regulamentações comerciais e processos de modo transparente o que implica simplificação e clareza dos processos e torná-los acessíveis ao maior número possível de empresas e indivíduos, aumenta-se a chance de negociar e aproveitar as oportunidades do mercado global. Assim, a facilitação de comércio pode ser tratada como meio de alterar as circunstâncias de comércio dentro de um país que dá origem a desigualdades de oportunidades ao comércio.

Mesmo que a literatura empírica não seja tão vasta, há crescente reconhecimento da fundamental importância de se facilitar o comércio com vistas a ampliar o comércio internacional, acelerar o crescimento e melhorar o bem-estar. Além disso, tem-se dado maior atenção às políticas, instituições e regulamentações adotadas no interior das fronteiras.

\footnotetext{
$1 \quad$ Os procedimentos de fronteira são compostos de vários estágios, sendo demasiadamente burocráticos. Estes tendem a ser nocivos ao comércio, pois estabelecem significativos custos de informação para potenciais importadores e exportadores (SOUZA; BURNQUIST, 2011). Engloba a administração alfandegária, eficiência portuária, normas ligadas a procedimentos comerciais, etc.

3 Inclui o ambiente regulatório, infraestrutura de transporte e portos, acesso a financiamentos, etc.
} 
As medidas destinadas a reduzir o custo da exportação na fronteira e por trás dela podem causar impacto significativo no comércio. Duval e Utoktham (2009) mostram que a melhoria do ambiente interno de negócios tem impacto positivo sobre a competitividade das exportações e que, no caso da Ásia, o ganho proveniente da melhoria no ambiente de negócios poderia gerar aumento adicional médio de 3\%, nas exportações bilaterais para os países da região. Além disso, o desempenho das exportações é afetado pelas características das empresas e por variáveis políticas, pois países que possuem comércio restritivo, regulamentos e administração aduaneira pobres não são atraentes para as empresas industriais. No caso de muitos países em desenvolvimento, é necessário um tempo relativamente longo para as exportações e importações se desfazerem dos procedimentos aduaneiros, o que leva, muitas vezes, ao pagamento de propina a funcionários para que esse tempo se reduza (CLARKE, 2005).

Apesar dos esforços para a redução das barreiras, alguns países ainda continuam a adotar tarifas e barreiras não tarifárias substanciais, o que, somado a outras fontes de custos comerciais, como infraestrutura pobre, torna o comércio cada vez mais restritivo, diminuindo a sua capacidade de participar do mercado internacional.

Estudos recentes demonstram ainda que as instituições nacionais e a infraestrutura disponível afetam diretamente os volumes de comércio entre os países (LIMÃO; VENABLES, 2001; WILSON; MANN; OTSUKI, 2003; FRANÇOIS; MANCHIN, 2006). Em geral, a literatura apoia a hipótese de que os custos do comércio doméstico e do ambiente econômico, além da qualidade do ambiente regulatório vigente, são determinantes significativos do volume de comércio entre os países.

Se diferentes fatores e políticas por trás da fronteira (behind the border) são inerentes ao desenvolvimento dos países e importantes para o comércio internacional, deve-se considerá-los nos modelos de comércio. Assim, é necessário olhar além das questões de fronteira, ou seja, atentar também para os fatores internos ao ambiente de negócios.

As medidas de simplificação de comércio estão relacionadas com a implementação de atividades, práticas e protocolos que visam melhorar procedimentos comerciais e aduaneiros (fracos e ineficientes) e, também, ações que busquem reduzir o contrabando, corrupção, fraudes, cobrança de impostos, falta de funcionários e falta de segurança na alfândega. O objetivo é mitigar as perdas que as empresas sofrem com problemas ligados à entrega de mercadorias, ausência de transparência e previsibilidade, exigências de documentação complexas (burocracia), procedimentos aduaneiros obsoletos, etc.

Espera-se que as medidas de simplificação de comércio possam beneficiar preferencialmente os países em vias de desenvolvimento, pois nestes as ineficiências ligadas ao comércio internacional têm custo mais elevado para as indústrias, 
comparadas às barreiras tarifárias. Entretanto, mesmo diante dessa perspectiva, esses países podem não ser estimulados a se comprometer com acordos multilaterais de facilitação de comércio. Entre os argumentos contrários está, por exemplo, o de que não há recursos suficientes para atualizar seus procedimentos aduaneiros com a adoção de padrões tecnológicos mais modernos.

Nesse sentido, este estudo procura trazer contribuições para as discussões sobre o tema avaliado, bem como os efeitos que variáveis internas dos países têm sobre os fluxos de comércio internacional de bens. Propõe-se uma definição ampliada do conceito de facilitação de comércio que considera a contribuição de indicadores de competitividade internos e dos de fronteira das nações sobre os fluxos de comércio internacional.

A intenção de analisar o impacto de melhorias nos fatores internos dos países sobre o comércio internacional é importante, dada a influência que esses avanços podem acarretar nas distintas variáveis econômicas e sociais dos países. Apesar dos esforços nas questões acerca da facilitação de comércio, grande parte dos trabalhos tem explorado somente fatores ligados à fronteira das nações. No entanto, nota-se que há percepção da importância das variáveis internas ao ambiente de negócio dos países como determinante do comércio internacional. Ainda que atualmente os trabalhos que exploram a temática da facilitação do comércio tenham ganhado força, estudos que utilizam os fatores internos dos países por meio dos indicadores aqui estudados não foram encontrados. Assim, neste estudo se procura verificar como a diferença nos indicadores internos de competitividade relativa afeta os fluxos comerciais.

Além desta introdução, este trabalho está dividido em mais quatro seções: na segunda apresenta-se uma discussão sobre a facilitação de comércio; na terceira faz-se uma exposição da metodologia utilizada; na quarta são apresentados e discutidos os resultados encontrados; e, por fim, na quinta traçam-se as considerações finais.

\section{A Importância de Fatores Internos e da Facilitação de Comércio sobre os Fluxos de Comércio Internacional}

A literatura sobre facilitação do comércio tem estimulado a pesquisa empírica das relações entre o comércio e os fatores que impedem o seu desenvolvimento, isto é, fatores que afetam os custos de comercialização internacional e, consequentemente, os fluxos de comércio. Com a redução das barreiras comerciais tradicionais, como as tarifas e quotas, voltou-se a atenção, principalmente, para os outros custos do comércio internacional, como os custos de transporte, das barreiras não tarifárias e dos outros fatores que afetam o comércio. A partir daí, as questões acerca das medidas de facilitação de comércio tornaram-se re- 
levantes nos estudos de comércio internacional. Em termos gerais, essa literatura sugere que as medidas de simplificação de comércio e um ambiente favorável de negócios existente nos diferentes países apresentam efeitos expressivos sobre o desenvolvimento do comércio.

A capacidade dos países de produzir e abastecer competitivamente um produto de interesse para as outras nações é essencial. A capacidade produtiva de um país é, sem dúvida, determinada em grande parte pelas suas políticas "por trás da fronteira" (ambiente doméstico), e, em particular, nas economias de mercado essas políticas estão diretamente relacionadas com o desenvolvimento do setor de negócios.

No contexto da facilitação do comércio, cujo foco está na racionalização de procedimentos, isso implica necessidade de os responsáveis pelas decisões políticas olharem para além da fronteira, no que tange aos procedimentos comerciais e às regulamentações. Em particular, a existência de um quadro comercial e de negócios coerentes e integrados de regulamentações pode ser decisivo para melhorar a competitividade das exportações.

Cabe destacar que a definição da facilitação de comércio ainda não ganhou consenso na literatura, mas, de modo simples, pode-se dizer que está ligada a políticas que levam à diminuição dos custos de importação e exportação (SOUZA, 2009). Além dessa definição simples, há outra conceituação que é mais comumente usada e que a descreve como a adoção de métodos que normalmente implicam maior eficiência na administração, nos processos e na logística de portos e alfândegas. No entanto, se for levada em conta uma visão mais ampla, pode-se incluir também o ambiente regulatório, questões relacionadas com harmonização mais intensa das normas e de regulamentos internacionais (WOO; WILSON, $2000^{4}$ apud WILSON; MANN; OTSUKI, 2003).

Apesar de iniciativas como a melhoria da infraestrutura de transportes, da liberalização do comércio e da promoção comercial, que, de certa forma, facilitam o comércio, elas não constituem ainda o que hoje é conhecido como facilitação do comércio. Em vez disso, a definição de facilitação de comércio envolve a ideia de redução de todos os custos de transação associados à execução, regulação e administração de políticas comerciais. Não obstante, aponta-se para uma tendência de mudança do significado e das implicações da facilitação do comércio há algumas décadas. A facilitação do comércio em um passado recente girava em torno das tentativas de harmonizar os regimes diferentes ou estabelecer elementos de reconhecimento mútuo entre diferentes taxas alfandegárias e regimes políticos afins. Atualmente, as convenções da OMC e de outras organizações mundiais estabelecem um conjunto comum de padrões internacionais ou usos de boas práticas

4 WOO, Y. P.; WILSON, J. S. Cutting through red tape: new directions for APEC's trade facilitation agenda. Asia Pacific Foundation of Canada. Vancouver: APEC, 2000. 
para todos os países. Assim, o desafio atual vai além de uma implementação e convergência dos produtores e operações aduaneiras com base nas normas internacionais. De um ponto de vista da facilitação do comércio, os regimes alfandegários assimétricos criam incerteza e, portanto, custos para os comerciantes internacionais (STAPLES, 2002).

Acredita-se que a facilitação do comércio tenha potencial para reduzir o custo de transação comercial e, assim, abaixar o preço das mercadorias comercializadas (HAMMAR, 2009). No entanto, vale ressaltar que nos países em desenvolvimento esses processos representam um desafio maior do que nos países desenvolvidos, já que a experiência mostra que o processo de implementação de programas de modernização das alfândegas pode ser lento. Assim, questões como assistência técnica e financeira podem afetar diretamente o desempenho de uma reforma aduaneira nos países em desenvolvimento. Ao pôr em prática processos que levem à simplificação e melhoria dos procedimentos aduaneiros, é possível que se criem nos países em desenvolvimento novas oportunidades, tanto de investimento quanto de comércio. A modernização das alfândegas é uma das ações que auxiliam na inclusão de um maior número de países em desenvolvimento no comércio internacional, principalmente em setores que produzem componentes industriais intermediários e que são sensíveis ao tempo (ENGMAN, 2005).

Os resultados do trabalho de Duval e Utoktham (2010) indicam que melhorias do ambiente de negócios nacionais podem ter impacto sobre a competitividade das exportações em uma dimensão análoga às medidas de facilitação do comércio e transporte. Além disso, os autores concluem que a competência de um país para o comércio é expressivamente comprometida pelas ações por trás das fronteiras e pelo ambiente de negócios do país importador. As reduções dos custos associados com políticas internas podem trazer maior retorno do que novas reduções de tarifas e de medidas não tarifárias, ou, mesmo, do que a busca de preferências comerciais suplementares (HOEKMAN; NICITA, 2008).

Os custos de transporte, por exemplo, são significativamente aumentados pela infraestrutura precária e por características geográficas adversas. Desse modo, os custos reais do comércio são importantes determinantes da capacidade de um país participar plenamente da economia mundial. A distância e a pobre infraestrutura nacional inibem a participação de muitas economias no comércio internacional (LIMÃO; VENABLES, 2001). As características regulatórias e institucionais também afetam as empresas que operam no ambiente nacional, fazendo com que em alguns países em desenvolvimento as barreiras à entrada sejam fontes adicionais de custos ao comércio (ARVIS et al., 2012). Portanto, é importante destacar que reformas em áreas como infraestrutura, regulamentação do setor de serviços e desenvolvimento do setor privado podem gerar benefícios significativos aos países em termos de redução dos custos comerciais. As particularidades pre- 
sentes nos países em desenvolvimento provocam externalidades negativas sobre as transações privadas e aumentam os custos comerciais, gerando efeitos negativos sobre o comércio e o crescimento econômico (IWANOW; KIRKPATRICK, 2007).

É nesse sentido que este trabalho busca ir além da análise de elementos de fronteira, procurando dar ênfase a elementos internos que afetam a competitividade dos países sobre os seus fluxos de comércio. Diante do apresentado, o desempenho dos negócios por trás da fronteira, neste estudo, é caracterizado por indicadores de competitividade, relacionados com áreas consideradas de particular importância para o desenvolvimento do comércio, como os indicadores do desempenho econômico geral dos países, a eficiência dos governos, a eficiência dos negócios e a infraestrutura.

\section{Aspectos Metodológicos e Especificação do Modelo Gravitacional}

\subsection{Modelo Gravitacional}

Para verificar os impactos que melhorias no ambiente doméstico de negócios possuem sobre os fluxos de comércio (melhorias nas variáveis que compõem os indicadores de competitividade), optou-se pelo uso do modelo gravitacional. Tal modelo tem-se mostrado apropriado para moldar os fluxos de comércio e, por isso, tem sido ferramenta básica para os economistas que buscam estudar a economia internacional (FEENSTRA, 2004). A inspiração desse modelo surgiu a partir da teoria da gravidade de Newton, mais especificamente, da analogia que é feita com a Lei da Gravitação Universal de 1687. Já na década de 1860, de acordo com Cheng e Wall (2005), Henry Carey aplicou pela primeira vez a teoria de Newton para analisar o comportamento humano, o que, por sua vez, levou à ampla utilização da equação de gravidade nas ciências sociais. Dentro do campo da economia, esses modelos tiveram sucesso empírico ao explicar várias questões referentes aos fluxos comerciais, tanto internacionais quanto inter-regionais.

Ao se analisar o comércio internacional, a premissa sob a qual o modelo de gravidade se baseia postula que o volume de comércio entre dois países é uma função crescente de suas rendas (num contexto em que o tamanho da economia de cada país é utilizado como uma proxy da renda) e uma função decrescente da distância entre os países (utilizada como proxy dos custos de transporte entre os países).

Conforme Cheng e Wall (2005), Tinbergen $(1962)^{5}$ e Pöyhönen $(1963)^{6}$ desenvolveram de forma independente um modelo de gravidade visando explicar

5 TINBERGEN, J. Shaping the world economy: suggestions for an international economic policy. New York: The Twentieth Century Fund, 1962.

6 PÖYHÖNEN, P. A. Tentative model for the volume of trade between countries. Weltwirtschaftliches Archiv, v. 90, n. 1, p. 93-100, 1963.

PAULA, J. S.; SILVA, O. M. Fatores Internos como Determinantes da Competitividade... 
o comércio bilateral. Na sua forma fundamental, assumiram que o valor do comércio entre os países aumentava com seu tamanho econômico, medido pela sua renda nacional, e diminuía com os custos de transporte entre eles, medido pela distância entre seus centros econômicos. Na sua forma mais simples, a equação da gravidade para o comércio pode ser representada da seguinte forma:

$$
\mathrm{T}_{\mathrm{ij}}=\alpha_{0} \mathrm{Y}_{\mathrm{i}}^{\alpha 1} \mathrm{Y}_{\mathrm{j}}^{\alpha 2} \mathrm{D}_{\mathrm{ij}}^{\alpha 3}
$$

em que $\alpha_{0}, \alpha_{1}, \alpha_{2}$ e $\alpha_{3}$ são parâmetros a ser estimados; $T_{i j}$ denota o fluxo de comércio do país i para o país j; $\mathrm{Y}_{\mathrm{i}}$ e $\mathrm{Y}_{\mathrm{j}}$ são o PIB dos países; e $\mathrm{D}_{\mathrm{ij}}$ é a variável distância, que inclui todos os fatores que possam criar resistência ao comércio.

Em 1966, Linnemann (1966) ${ }^{7}$ incluiu a população no modelo básico, como uma medida adicional da dimensão do país, empregando o que se conhece como modelo de gravidade aumentado (CHENG; WALL, 2005).

O trabalho de Anderson (1979) foi, contudo, o primeiro a fornecer microfundamentos para o modelo em questão. O que o autor propôs, basicamente, foi uma explicação teórica para a equação da gravidade que tinha como contexto a análise do comércio de commodities.

Posteriormente, uma extensão proposta por Anderson e Wincoop (2004) torna-se a abordagem teórica que mais tem sido empregada para dar suporte ao modelo gravitacional. Esses autores oferecem importante contribuição ao ressaltar que os fluxos comerciais entre pares de países são diretamente proporcionais ao seu PIB e inversamente proporcionais às barreiras comerciais relativas. Assim sendo, fatores como a localização geográfica de um país é assim capaz de afetar a sua competitividade no comércio internacional. Além disso, o modelo é especificado de modo a possibilitar análises setoriais. A equação gravitacional teórica com base nesses autores é representada da seguinte forma:

$$
\ln X_{i j}^{k}=\ln E_{j}^{k}+\ln Y_{i}^{k}-\ln Y^{k}+\left(1-\alpha_{k}\right) \ln t_{i j}^{k}\left(1-\alpha_{k}\right) \ln P_{j}^{k}-\left(1-\alpha_{k}\right) \ln \pi_{i}^{k}
$$

em que $\mathrm{X}_{\mathrm{ij}}^{\mathrm{k}}$ representa as exportações do setor $k$ da região (ou país) i para a região j; $E_{j}^{k}$ é o dispêndio do país j com o grupo de produtos $k$; $Y_{i}^{k}$ e $Y^{k}$ são, respectivamente a produção do país $i$ e a produção agregada (mundial) no setor $k ; t_{i j}{ }^{k}$ representa uma função dos custos de comercialização; e $\mathrm{P}_{\mathrm{j}}^{\mathrm{k}} \mathrm{e} \pi_{\mathrm{i}}^{\mathrm{k}}$ representam índices de preços, sendo também identificados como índices de resistência multilateral.

De acordo com Shepherd e Wilson (2008), o modelo teórico de Anderson e Wincoop pode ser adotado como a abordagem padrão. Trata-se de um modelo de análise ex post, que tem sido utilizado para verificar a magnitude e os efeitos de

$7 \quad$ LINNEMANN, H. An econometric study of international trade flows. Amsterdam: North-Holland Publishing Company, 1966. 
diversas variáveis sobre os fluxos comerciais, tais como a volatilidade cambial, o impacto da aplicação de tarifas, a mobilidade da mão de obra, diversos custos de transporte, etc.

Deve-se ressaltar que a notoriedade do modelo de Anderson e Wincoop (2004) se deve, principalmente, a três fatores. O primeiro decorre da convicção de que os fluxos de comércio internacional são um elemento chave em todos os tipos de relações econômicas, o que, por sua vez, implica conhecimento de como os fluxos comerciais deveriam se comportar. O segundo fator aponta para a facilidade quanto aos dados necessários para estimação do modelo. Por fim, tem-se uma série de aplicações empíricas de grande visibilidade que têm estabelecido respeitabilidade aos modelos de gravidade. Além disso, estabelecem um grupo de práticas comuns que têm sido usadas para lidar com as escolhas empíricas ad hoc, com quais os pesquisadores se deparam, ou seja, são estabelecidas práticas padrão que facilitam o trabalho dos pesquisadores (BALDWIN; TAGLIONI, 2006). Segundo Cheng e Wall (2005), pode-se destacar também, como um elemento de sucesso desse modelo, as suas propriedades econométricas, cujo poder de explicação empírica traduz-se simplesmente por meio de um coeficiente de ajustamento relativamente elevado.

O modelo de gravidade tem sido utilizado para estimar o impacto de diversas questões políticas, incluindo acordos de comércio regional, uniões monetárias, blocos políticos, direitos de patentes, fatores institucionais, etc. Nesses estudos tem sido comum adicionar outras variáveis para melhor refletir as condições da realidade. No caso presente, além das variáveis básicas do modelo gravitacional (PIB e distância geográfica entre países), são incorporados as tarifas e um conjunto de indicadores de competitividade. A especificação funcional proposta para a equação de gravidade está expressa na equação 3:

$$
\begin{array}{r}
\ln \left(\mathrm{M}_{\mathrm{ij}, \mathrm{t}}\right)=\alpha_{\mathrm{i}}+\delta_{\mathrm{j}}+\gamma_{\mathrm{t}}+\beta_{1} \ln \left(\mathrm{Y}_{\mathrm{i}, \mathrm{t}}\right)+\beta_{2} \ln \left(\mathrm{Y}_{\mathrm{j}, \mathrm{t}}\right)+\beta_{3} \ln \left(\mathrm{D}_{\mathrm{i}, \mathrm{j}}\right)+\beta_{4} \ln \left(1+\tau_{\mathrm{ij}, \mathrm{t}}\right)+ \\
\beta_{5}\left(\mathrm{DECO}_{\mathrm{i}, \mathrm{t}}\right)+\beta_{6}\left(\mathrm{EGov}_{\mathrm{i}, \mathrm{t}}\right)+\beta_{7}\left(\mathrm{ENeg}_{\mathrm{i}, \mathrm{t}}\right)+\beta_{8}\left(\operatorname{Infra}_{\mathrm{i}, \mathrm{t}}+\varepsilon_{\mathrm{ij}, \mathrm{t}}\right.
\end{array}
$$

em que $M_{\mathrm{i}, \mathrm{t}}$ indica o valor das importações realizadas pelo país $i$ do país j no ano $t ; \alpha_{\mathrm{i}}$ e $\delta_{\mathrm{j}}$ representam os efeitos fixos para exportadores (i) e importadores (j) invariantes no tempo; $\gamma_{\mathrm{t}}$ representa as variáveis binárias para os anos da amostra; $Y_{i, t} \mathrm{e}$ $Y_{j, t}$ são, respectivamente, o PIB do país importador (i) e do país exportador (j) no tempo $t ; D_{i, j}$ é a distância do país $i$ ao país $j ;\left(1+\tau_{i \mathrm{ij}, t}\right)$ é a tarifa média aplicada pelo país $i$ às importações do país $j$ no ano $t ; D E c O_{i, t}$ representa o fator que mede o desempenho econômico do país i no ano $t ; E G o v_{i, t}$ representa o fator que mensura a eficiência do governo no país i no ano $t ; E_{N e g_{i, t}}$ representa o fator que mede a eficiência dos negócios do país i no ano $t$; Infra $a_{i, t}$ é o indicador da infraestrutura do país i no ano t; e $\varepsilon_{\mathrm{ij}, t}$ corresponde ao termo de erro aleatório. 
Para o modelo gravitacional proposto na equação 3, utiliza-se um painel com 59 países, ${ }^{8}$ para os anos entre 1997 e 2011, estimado pelos métodos de efeitos fixos, efeitos aleatórios e também pelo método Poisson pseudo-maximum likelihood (PPML).

A adequação e a robustez dos resultados são aferidas por diferentes estatísticas. A significância dos coeficientes do modelo de efeitos fixos é examinada por meio do teste $\mathrm{F}$ de Chow (GREENE, 2003). A presença de efeitos individuais é verificada com o teste do multiplicador de Lagrange, de Breusch e Pagan (GUJARATI, 2011). Também emprega-se o teste de Hausman para verificar se as diferenças nos coeficientes não são sistemáticas. O modelo proposto é estimado também pelo método PPML. A utilidade de tal método está no fato deste apresentar, usualmente, estimativas mais consistentes na presença de heterocedasticidade e devido à existência de fluxos bilaterais com valores iguais a zero (missing) na base de dados (SOUZA; BURNQUIST, 2011). A diferença nesse caso é que a variável dependente será expressa em nível.

Segundo Santos Silva e Tenreyro (2006), muitas aplicações econométricas têm ignorado o fato de que a interpretação dos parâmetros do modelo log-linear, estimado por mínimos quadrados ordinários $(\mathrm{MQO})$, pode ser enganosa na presença de heterocedasticidade. Além disso, apontam que a existência de observações zero da variável dependente cria um problema adicional para o uso da forma log-linear na equação de gravidade. Através de testes, os autores mostram que a heterocedasticidade é realmente um problema grave tanto na equação de gravidade tradicional proposta por Tinbergen (1962), quanto na forma mais recente sugerida por Anderson e Wincoop (2003).

\subsection{Fonte e Tratamento de Dados}

O modelo proposto na equação 3 é estimado com base em um painel com 59 países (ver Figura 1), selecionados no Anuário da Competitividade Mundial (INTERNATIONAL INSTITUTE OF MANAGEMENT AND DEVELOPMENT, 2011), para os anos compreendidos entre 1997 e 2011. Nessa equação, a medida do fluxo de comércio refere-se ao valor da importação bilateral, retirada da base de dados do World Integrated Trade Solution (Wits), tendo como fonte, dados do Uncomtrade. Na Tabela 1 mostram-se detalhes sobre as variáveis utilizadas.

$8 \quad$ Os países incluídos na amostra são: África do Sul, Alemanha, Argentina, Austrália, Áustria, Bélgica, Brasil, Bulgária, Canadá, Catar, Cazaquistão, Chile, China, Cingapura, Colômbia, Coreia, Croácia, Dinamarca, Emirados Árabes Unidos, Eslováquia, Eslovênia, Espanha, Estônia, Estados Unidos, Filipinas, Finlândia, França, Grécia, Holanda, Hong Kong, Hungria, Índia, Indonésia, Irlanda, Islândia, Israel, Itália, Japão, Jordânia, Lituânia, Luxemburgo, Malásia, México, Noruega, Nova Zelândia, Peru, Polônia, Portugal, Reino Unido, República Tcheca, Romênia, Rússia, Suécia, Suíça, Tailândia, Taiwan, Turquia, Ucrânia e Venezuela. 
Tabela 1 - Variáveis utilizadas, fontes de dados, sinais esperados e descrição

\begin{tabular}{|c|c|c|c|}
\hline Variável & Fonte & $\begin{array}{c}\text { Sinal } \\
\text { esperado }\end{array}$ & Descrição \\
\hline $\mathrm{M}_{\mathrm{ij}, \mathrm{t}}$ & Wits & & $\begin{array}{l}\text { Importação bilateral entre o país impor- } \\
\text { tador e o parceiro comercial (em } 1.000 \\
\text { dólares). }\end{array}$ \\
\hline $\mathrm{D}_{\mathrm{i}, \mathrm{j}}$ & CEPII & - & $\begin{array}{l}\text { Distância entre dois países com base nas } \\
\text { distâncias bilaterais entre as maiores cidades } \\
\text { dos dois países (em km). }\end{array}$ \\
\hline$Y_{i, t} / Y_{j, t}$ & IMD & + & $\begin{array}{l}\text { PIB, a preços correntes (em bilhões de } \\
\text { dólares americanos). }\end{array}$ \\
\hline$\tau_{\mathrm{ij}, \mathrm{t}}$ & Wits & - & $\begin{array}{l}\text { Tarifa de importação efetiva ponderada } \\
\text { pelo comércio aplicada pelo importador } \\
\text { sobre o parceiro. }\end{array}$ \\
\hline $\mathrm{DECo}_{\mathrm{i}, \mathrm{t}}$ & IMD & + & $\begin{array}{l}\text { Avaliação macroeconômica da economia } \\
\text { doméstica. }\end{array}$ \\
\hline $\mathrm{EGov}_{\mathrm{i}, \mathrm{t}}$ & IMD & + & $\begin{array}{l}\text { Em que medida as políticas do governo são } \\
\text { favoráveis para a competitividade. }\end{array}$ \\
\hline $\mathrm{ENeg}_{\mathrm{i}, \mathrm{t}}$ & IMD & + & $\begin{array}{l}\text { Em que medida as empresas estão realizan- } \\
\text { do de forma inovadora, rentável e respon- } \\
\text { sável os seus negócios. }\end{array}$ \\
\hline Infra $_{i, t}$ & IMD & + & $\begin{array}{l}\text { Até que ponto os recursos tecnológicos, } \\
\text { científicos e humanos satisfazem as necessi- } \\
\text { dades dos negócios. }\end{array}$ \\
\hline $\operatorname{Dsn}_{\mathrm{i}, \mathrm{j}}$ & OMC & + & $\begin{array}{l}\text { Variável dummy que assume o valor "0" se } \\
\text { os dois países são classificados como do Sul } \\
\text { ou do Norte e "1" caso contrário, quando } \\
\text { um país é classificado como Sul e o outro } \\
\text { como Norte. }\end{array}$ \\
\hline
\end{tabular}

Fonte: Elaboração própria.

Nota: CEPII - Centre d'Etudes Prospectives et d'Information Internationales. IMD - International Institute for Management Development. WITS - World Integrated Trade Solutions.

Os valores do PIB foram obtidos do Banco Mundial e os valores para a distância entre os países foram retirados da base de dados do CEPII (Centre d'Études Prospectives et d'Informations Internationales). O valor das tarifas foi obtido do Wits, baseado em dados da OMC. Na estimação do modelo proposto, utilizou-se o programa Stata 12.0. Como facilitadores de comércio, foram utilizados indicadores de competitividade relativa dos países. Esses indicadores estão disponíveis no Anuário de Competitividade Mundial do IMD (INTERNATIONAL..., 2011), que analisa 
o desempenho dos países com base em 331 critérios para medir as diferentes faces da competitividade.

O ambiente nacional é dividido em quatro fatores principais, que são desempenho econômico geral, eficiência dos governos, eficiência dos negócios e infraestrutura. Cada um desses quatro fatores é subdividido em cinco subfatores, contendo diferentes números de critérios, conforme mostrado na Tabela 2.

$\mathrm{O}$ argumento para o uso de um número diferente de critérios para cada subfator baseia-se no fato de que, por exemplo, um número menor de critérios é necessário para avaliar os preços dos produtos do que para avaliar o nível da educação em cada país. Porém, cada subfator, a despeito do número de critérios que possua, recebe o mesmo peso na consolidação global dos resultados, que é de $5 \%$. No final, cada fator de competitividade terá um valor entre 0 e 100. A partir da agregação dos resultados dos 20 subfatores, cada um com peso de $5 \%$, é que se obtém uma classificação geral sobre a competitividade dos países (INTERNATIONAL..., 2011).

Tabela 2 - Fatores de competitividade e critérios

\begin{tabular}{|c|c|c|c|c|}
\hline Fatores & $\begin{array}{l}\text { Desempenho } \\
\text { econômico } \\
\text { (78 critérios) }\end{array}$ & $\begin{array}{l}\text { Eficiência do } \\
\text { governo } \\
\text { (71 critérios) }\end{array}$ & $\begin{array}{l}\text { Eficiência dos } \\
\text { negócios } \\
\text { (68 critérios) }\end{array}$ & $\begin{array}{l}\text { Infraestrutura } \\
\text { (114 critérios) }\end{array}$ \\
\hline \multirow{5}{*}{ 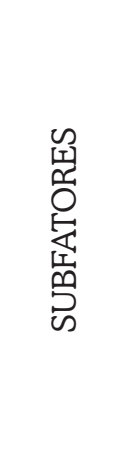 } & $\begin{array}{l}\text { Economia } \\
\text { doméstica }\end{array}$ & $\begin{array}{l}\text { Finanças } \\
\text { públicas }\end{array}$ & Finanças & Educação \\
\hline & $\begin{array}{l}\text { Comércio } \\
\text { internacional }\end{array}$ & $\begin{array}{l}\text { Quadro } \\
\text { institucional }\end{array}$ & $\begin{array}{l}\text { Práticas de } \\
\text { gestão }\end{array}$ & $\begin{array}{l}\text { Infraestrutura } \\
\text { básica }\end{array}$ \\
\hline & $\begin{array}{l}\text { Investimento } \\
\text { internacional }\end{array}$ & $\begin{array}{l}\text { Legislação } \\
\text { comercial }\end{array}$ & $\begin{array}{l}\text { Produtividade } \\
\text { e eficiência }\end{array}$ & $\begin{array}{l}\text { Infraestrutura } \\
\text { tecnológica }\end{array}$ \\
\hline & Emprego & Política fiscal & $\begin{array}{l}\text { Mercado de } \\
\text { trabalho }\end{array}$ & $\begin{array}{l}\text { Infraestrutura } \\
\text { científica }\end{array}$ \\
\hline & Preços & Quadro social & $\begin{array}{l}\text { Atitudes e } \\
\text { valores }\end{array}$ & $\begin{array}{l}\text { Saúde e meio } \\
\text { ambiente }\end{array}$ \\
\hline
\end{tabular}

Fonte: International Institute of Management and Development (2011).

\section{Análise dos Resultados}

Neste estudo procurou-se verificar principalmente como a diferença nos indicadores de competitividade relativa afeta os fluxos comerciais. Sendo assim, nesta parte do texto apresentam-se o cenário da competitividade dos países e os resultados do exercício econométrico proposto. 


\subsection{Panorama Geral da Competitividade Mundial}

Em termos continentais observou-se que entre 2007 e 2011 os países da amostra tiveram um comportamento semelhante ao longo dos anos. Os países desenvolvidos da América do Norte estão entre os mais competitivos do mundo, com exceção do México. No caso dos países em desenvolvimento, o destaque está no desempenho do Chile, que tem como atrativo a sua estabilidade política, o seu dinamismo econômico e um baixo índice de corrupção. Na Ásia, o desempenho competitivo de destaque vai para Hong Kong e Singapura, e para Taiwan, Malásia e Catar nos anos recentes. No caso do continente europeu, Suíça, Suécia, Alemanha, Dinamarca, Luxemburgo, Noruega e Holanda apresentam um bom desempenho competitivo nos últimos anos.

Entre 1997 e 2011, entre os países desenvolvidos, Itália, Grécia, Polônia e Rússia apresentaram índice global de competitividade abaixo da média geral. A incerteza refletida no risco sistêmico da zona do euro tem sido amenizada principalmente por promessas de reformas fiscais. Entretanto, a conjuntura econômica nessa região tem se deteriorado, sobretudo na Itália, Espanha e Grécia, que são países que apresentam como pontos fracos o mercado de trabalho e a política fiscal. Isso, por sua vez, tem como consequência tendências negativas nos fatores que incorporam os indicadores de competitividade. Por outro lado, países em desenvolvimento como Chile, China, República Checa, Hong Kong, Hungria, Islândia, Israel, Coreia, Malásia, Singapura, Taiwan e Tailândia tiveram desempenho superior à média encontrada. Observa-se que, por sua vez, esses países apresentam como pontos fortes mais notáveis justamente a eficiência de seu mercado de trabalho e da política fiscal.

Na Figura 1 é ilustrado o índice total de competitividade médio. Observase que, de 1997 a 2011 em média, estão entre os dez países mais competitivos Estados Unidos, Singapura, Hong Kong, Luxemburgo, Suíça, Canadá, Dinamarca, Holanda, Finlândia e Austrália. De acordo com o IMD, entre os fatores que propiciam a maior competitividade dessas nações estão a elevada produtividade da força de trabalho, o acesso a financiamento, o dinamismo econômico, a infraestrutura confiável e o elevado nível educacional, entre outros. Entre os Brics (Brasil, Rússia, Índia, China e África do Sul), apenas a China obteve um desempenho superior à média, que em grande parte se deve ao alto dinamismo existente na sua economia. A África do Sul, apesar de ter um baixo desempenho se comparado aos demais países, apresentou um comportamento ascendente ao longo dos anos. 
Figura 1 - Índice total médio de competitividade (1997-2011)

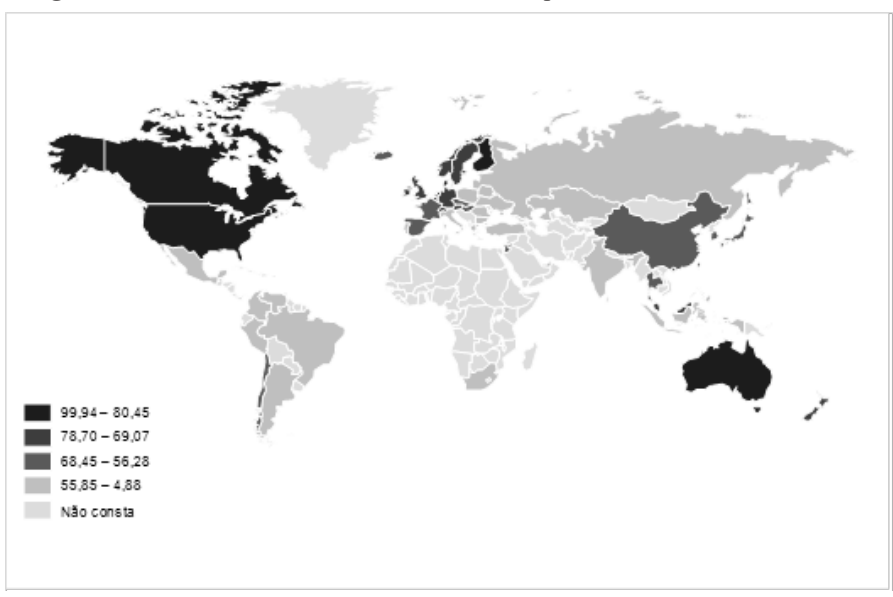

Fonte: Elaboração própria a partir de dados da pesquisa.

Quando analisados os indicadores de competitividade em termos desagregados, temos que, entre os países desenvolvidos, apenas a Rússia apresentou o índice de desempenho econômico abaixo da média nos 15 anos de análise. De outro lado, grande parte dos países em desenvolvimento esteve acima da média no indicador de desempenho econômico. Entre eles estão Brasil, Chile, China, Índia, Israel, Coreia, México, Taiwan, Tailândia, etc.

Para o indicador de eficiência do governo, assim como no caso do índice total, apresentaram índice abaixo da média: Itália (necessita combater a evasão fiscal e impor menores taxas de impostos sobre as empresas e o trabalho); Grécia (deve promover reformas institucionais para reduzir o tamanho do setor público e facilitar a saída da crise); Polônia (carece de reduções no déficit orçamental e da dívida pública); e Rússia (uma saída pode ser a diversificação das atividades econômicas para diminuir a dependência dos recursos naturais).

Os mesmos países em desenvolvimento que tiveram o índice global de competitividade acima da média também obtiveram o indicador de eficiência nos negócios superior à média, incluindo também Colômbia, Estônia, Índia, México e Filipinas. Itália, Polônia, Portugal e Rússia apresentaram valores abaixo da média para o indicador de eficiência nos negócios. O mesmo ocorreu para os seguintes países em desenvolvimento: Argentina, Bulgária, Colômbia, Croácia, Indonésia, Jordânia, Cazaquistão, Lituânia, México, Peru, Catar, Romênia, Eslováquia, Eslovênia, Emirados Árabes, Ucrânia e Venezuela.

Por fim, no caso do indicador de infraestrutura entre os países do Sul, apenas Chile, China, República Tcheca, Estônia, Hong Kong, Hungria, Islândia, Israel, Coreia, Malásia, Singapura, Eslovênia e Taiwan apresentaram valores superiores 
à média. Por outro lado, analisando os países desenvolvidos, somente Polônia e Rússia tiveram ao longo dos 15 anos valores abaixo da média geral.

\subsection{Resultados das Estimativas da Equação Gravitacional ${ }^{9}$}

Os resultados obtidos na estimação do modelo econométrico proposto são apresentados a seguir. Inicialmente o modelo é estimado apenas com o índice geral, que agrega os outros quatro indicadores. Posteriormente, são apresentados os resultados da estimação da equação gravitacional com os quatro indicadores de competitividade e, por fim, uma equação incluindo variáveis binárias para diferenciar o comércio entre os países do sul e do norte. Nesse último caso, a ideia é captar os efeitos do grau de desenvolvimento dos países nos fluxos de comércio.

Como as variáveis foram transformadas por logaritmo, seus coeficientes podem ser interpretados diretamente como elasticidades. A base de dados utilizada no estudo apresentou um número considerável de observações. Foram 15 anos de importações de 59 países, com 58 outros parceiros comerciais, totalizando 51.330 observações, das quais aproximadamente $12 \%$ apresentaram fluxo zero ou missing.

Por meio dos testes de Chow, Hausman e Breush-Pagan pode-se inferir que o modelo de efeitos fixos foi o mais adequado. Esse resultado está de acordo com as ideias de Baldwin e Taglioni (2006), que sugerem que as equações de gravidade devem ser estimadas com efeitos fixos, dada a presença de variáveis e características não observáveis no modelo, que são correlacionadas com termos de custos de comércio. Assim sendo, pode-se dizer que não é possível rejeitar a hipótese de que efeitos de heterogeneidades não observáveis dos países afetam o comércio bilateral. Ou seja, esse modelo é preferível uma vez que leva em consideração as idiossincrasias dos países. Adicionalmente, dada a já mencionada ausência de fluxos comerciais na amostra, estimou-se também a equação de gravidade proposta, por meio do método PPML.

De maneira geral, os resultados foram robustos, com alta significância estatística para os coeficientes estimados e com os coeficientes de determinação variando entre 0,39 e 0,90 . Os resultados do modelo estimado por PPML apresentaram, de modo geral, os sinais sugeridos pela teoria econômica.

\subsubsection{Modelo com o Índice Total}

Os resultados apresentados a seguir são para o modelo com a seguinte especificação funcional:

9 Para mais detalhes dos resultados das variáveis binárias para os anos da amostra, importador e exportador, ver Paula (2013). 


$$
\ln \left(\mathrm{M}_{\mathrm{ij}, \mathrm{t}}\right)=\alpha_{\mathrm{i}}+\delta_{\mathrm{j}}+\gamma_{\mathrm{t}}+\beta_{1} \ln \left(\mathrm{Y}_{\mathrm{i}, \mathrm{t}}\right)+\beta_{2} \ln \left(\mathrm{Y}_{\mathrm{j}, \mathrm{t}}\right)+\beta_{3} \ln \left(\mathrm{D}_{\mathrm{i}, \mathrm{j}}\right)+\beta_{4} \ln \left(1+\tau_{\mathrm{ij}, \mathrm{t}}\right)+\beta_{5}\left(\mathrm{It}_{\mathrm{i}, \mathrm{t}}\right)+\varepsilon_{\mathrm{ij}, \mathrm{t}}
$$

em que $\mathrm{It}_{\mathrm{i}, \mathrm{t}}$ representa o indicador geral de competitividade do país $i$ no ano $t$.

Em termos da magnitude, observou-se que os coeficientes estimados para o PIB dos países exportadores e importadores apresentaram-se positivos e estatisticamente significativos, ao nível de 1\%. De modo semelhante aos resultados encontrados na literatura, observou-se que variações positivas nessa variável geraram variações, também positivas, no comércio. No caso do modelo de efeitos fixos, por exemplo, as elasticidades-renda estimadas foram de aproximadamente 1,5 e 0,6 para os países importadores e exportadores, respectivamente, permitindo inferir que aumentos de $10 \%$ nas rendas levariam, em média, a aumentos nos fluxos de importação em 15\% e 6\% (ver Tabela 3).

Os coeficientes estimados para a distância apresentaram sinais negativos em todos os casos, conforme esperado, sendo estatisticamente significativos. Isso indica que quanto maior a distância entre os países, menor será o nível de comércio. A variável tarifa não apresentou sinal conforme o esperado no modelo de efeitos fixos, mas foi negativa e estatisticamente significativa no modelo PPML. Com pequenas exceções (por exemplo, a Rússia), a maioria dos países considerados é membro da OMC e já tinha reduzido suas tarifas após a Rodada Uruguai, ${ }^{10}$ fazendo com que elas apresentassem um efeito pequeno sobre os fluxos de comércio. No caso do modelo PPML, por exemplo, uma elevação de 10\% no valor das tarifas reduziria o comércio bilateral em somente $0,45 \%$.

Comparando os resultados do modelo de efeitos fixos e do PPML, notou-se que a elasticidade da distância é substancialmente maior no primeiro caso $(-1,22$ versus - 0,86), o que está de acordo com o sugerido pela literatura, de que a estimativa obtida com a utilização de PPML gera elasticidades inferiores às estimativas de MQO tradicionais (ARVIS et al., 2012).

10 A Rodada Uruguai corresponde a uma das diversas rodadas de negociações comerciais multilaterais estabelecidas no Acordo Geral sobre Tarifas e Comércio (GATT), que objetivaram principalmente a redução de tarifas e de barreiras não tarifárias. 
Tabela 3 - Resultados estimados para a equação com o índice total

\begin{tabular}{|c|c|c|c|c|}
\hline Variável & \multicolumn{2}{|c|}{ Efeitos fixos } & \multicolumn{2}{|c|}{ PPML } \\
\hline Lnpibi & $1,544 * * *$ & $1,5306 * * *$ & $0,760 * * *$ & $0,7842 * * *$ \\
\hline Lnpibj & $0,617^{* * *}$ & $0,6246 * * *$ & $0,650 * * *$ & $0,6488 * * *$ \\
\hline Lndistancia & $-1,220 * * *$ & $-1,2464 * * *$ & $-0,865^{* * *}$ & $-0,8733^{* * *}$ \\
\hline Lntarifa & $0,063 * * *$ & $0,0646 * * *$ & $-0,045 * * *$ & $-0,0495 * * *$ \\
\hline it & $-0,013 * * *$ & $-0,0192 * * *$ & $0,003 * *$ & $0,0059 * * *$ \\
\hline nsit & . & $0,0034^{* * *}$ & . & $0,0035 * * *$ \\
\hline snit & . & $0,0080 * *$ & . & $-0,0041 * *$ \\
\hline ssit & . & $0,0054^{*}$ & . & $-0,0027$ \\
\hline constante & $10,072 * * *$ & $10,3379 * * *$ & $12,463 * * *$ & $12,3440 * * *$ \\
\hline $\mathrm{R}^{2}$ & 0,6164 & 0,6171 & 0,6956 & 0,8928 \\
\hline $\mathrm{N}^{\circ}$ de Observações & 51330 & 51330 & 51330 & 51330 \\
\hline
\end{tabular}

Fonte: Elaboração própria.

Nota: " $\mathrm{p}<0.10$; *" $\mathrm{p}<0.05$; *** $\mathrm{p}<0.01$. Para o modelo de efeito aleatório é reportado o $\mathrm{R}^{2}$ "within".

A estimativa do parâmetro que mede o efeito do índice total de competitividade apresentou-se estatisticamente significativa nos dois modelos, contudo com o sinal trocado no modelo de efeitos fixos. No caso do modelo PPML, o resultado sugere que melhorias relativas nos fatores que compõem o índice geral de competitividade proporcionariam aumentos nos fluxos de comércio bilaterais. As estimativas obtidas pelo método PPML foram todas significativas e apresentaram os sinais sugeridos pela teoria. Mesmo gerando elasticidades inferiores às estimativas de MQO tradicionais, esse modelo parece ser o mais adequado pelo elevado coeficiente de ajustamento e por apresentar resultados mais robustos e semelhantes aos encontrados na literatura.

O parâmetro estimado pela dummy de interação fornece a diferença nos indicadores entre países desenvolvidos e em desenvolvimento. ${ }^{11}$ Assim, a especificação funcional para a equação de gravidade é

$$
\begin{aligned}
& \ln \left(\mathrm{M}_{\mathrm{ij}, \mathrm{t}}\right)=\alpha_{\mathrm{i}}+\delta_{\mathrm{j}}+\gamma_{\mathrm{t}}+\beta_{1} \ln \left(\mathrm{Y}_{\mathrm{i}, \mathrm{t}}\right)+\beta_{2} \ln \left(\mathrm{Y}_{\mathrm{j}, \mathrm{t}}\right)+\beta_{3} \ln \left(\mathrm{D}_{\mathrm{i}, \mathrm{j}}\right)+\beta_{4} \ln \left(1+\tau_{\mathrm{ij}, \mathrm{t}}\right)+\beta_{5}\left(\mathrm{It}_{\mathrm{i}, \mathrm{t}}\right)+\delta_{1} n s i t \\
& +\delta_{2} \operatorname{snit}+\delta_{3} s s i t+\varepsilon_{\mathrm{ij}, \mathrm{t}}
\end{aligned}
$$

em que ns é uma variável binária que assume valor 1 , caso o país importador $i$ seja classificado como país do norte e o país exportador j seja classificado como país do sul; sn é uma variável binária que assume valor 1, caso o país importador i

11 Lembra-se que neste trabalho definiu-se o norte como países de alta renda, ou desenvolvidos, e o sul como os demais países. 
seja classificado como país do sul e o país exportador j seja classificado como país do norte; ss é uma variável binária que assume valor 1, caso o par de países sejam ambos classificados como do sul, e 0 caso contrário. A categoria de controle, portanto, será o caso em que o par de países seja classificado como do norte.

Em conformidade com o apresentado anteriormente, os resultados foram semelhantes. A estimativa do índice de competitividade total apresentou-se estatisticamente significativa e, positiva, no modelo PPML. Assim, como visto anteriormente, isso sugere que melhorias relativas nos fatores que compõem o índice geral de competitividade teriam efeitos positivos sobre os fluxos de comércio bilateral.

No caso PPML, observa-se que as variáveis dummy nsit e snit tiveram seus coeficientes estatisticamente significativos. A variável binária nsit foi positiva, o que indica que, quando o país importador é um país considerado como do norte e o país exportador considerado como do sul, o importador terá vantagem no comércio, dada pela diferença de competitividade existente entre ele e seu parceiro. De modo oposto, quando o país importador é um país considerado como do sul e o país exportador do norte, o importador tem uma desvantagem no comércio, já que seu parceiro possui uma competitividade maior (sinal negativo de snit).

A variável binária ssit não apresentou significância estatística, de forma que não se pode inferir sobre o impacto da competitividade sobre o comércio sul-sul. Apesar de não significativa, o sinal negativo encontrado para o comércio sul-sul reforça o argumento de Arvis et al. (2012) de que os custos comerciais enfrentados no comércio sul-sul são muito mais elevados do que os custos comerciais que afetam o comércio norte-norte. Além disso, destacam que o comércio dentro ou entre grupos de renda do sul está sujeito a custos substancialmente mais elevados do que o comércio entre os países do norte.

\subsubsection{Modelo com Indicadores de Competitividade Desagregados}

Neste caso, a especificação funcional para a equação de gravidade segue a equação 3, apresentada anteriormente, com os resultados mostrados na Tabela 4.

Da mesma forma que no caso anterior, os coeficientes estimados para o PIB, tanto dos países importadores, quanto dos países exportadores, foram positivos e estatisticamente significativos ao nível de $1 \%$ de probabilidade. As estimativas dos coeficientes da distância apresentaram sinais negativos conforme esperado, sendo também estatisticamente significativas. Mais uma vez o coeficiente estimado para a tarifa foi negativo apenas no modelo PPML.

Quanto aos indicadores de competitividade, ${ }^{12}$ o coeficiente estimado para o desempenho econômico dos países apresentou sinal positivo conforme espera-

12 É necessário reconhecer que as variáveis explicativas representadas pelos indicadores podem ser potencialmente endógenas ao modelo. Muitas vezes, é intuitivo o uso de uma proxy que não 
do e significância estatística (exceto no modelo PPML). Já no caso do indicador de eficiência dos negócios, os coeficientes estimados apresentaram-se positivos e estatisticamente significativos. No modelo de efeitos fixos, tanto o indicador que mede a eficiência dos governos, quanto o que representa a infraestrutura dos países, apresentaram significância estatística, entretanto com sinais trocados. Já no modelo PPML essas variáveis não foram estatisticamente significativas.

Os resultados do modelo PPML são muito semelhantes aos anteriores. Quanto aos indicadores de competitividade, somente a eficiência do governo teve significância estatística e apresentou sinal esperado. Esse fator inclui áreas estratégicas para o desempenho dos fluxos comerciais, entre elas, o desempenho do mercado de trabalho doméstico, o grau de risco e o nível de transparência das instituições financeiras, a adaptabilidade das empresas ao ambiente nacional, entre outros. Assim, um bom desempenho dos países nessas áreas pode ser determinante para um desempenho comercial positivo.

No que tange às variáveis binárias de interação observou-se, por exemplo, que, para o caso em que o país importador é um país considerado como do sul e o país exportador considerado como do norte, há retornos positivos ao comércio, no que se refere à infraestrutura do importador, e negativos quanto ao desempenho econômico e à eficiência do governo. De modo oposto, quando o país importador é um país do norte e o país exportador é um do sul, há retornos negativos ao comércio no que se refere à infraestrutura do importador e positivo quanto ao desempenho econômico.

Observou-se, assim, que, quando os importadores do sul comercializam com os países do norte, investimentos em infraestrutura favorecem o comércio. De outro lado, quando países do norte importam do sul, investimentos em infraestrutura no norte desfavorecem o comércio. A explicação para isso pode estar no fato de que melhorias em infraestrutura nos países desenvolvidos podem favorecer o comércio norte-norte, ao invés do comércio norte-sul, já que, de acordo com Arvis et al. (2012), mesmo depois de vários avanços que diminuíram a distância (não somente geográfica) entre os países, esse ainda é um fator decisivo dos custos comerciais que envolvem os países do sul, quando comparados ao comércio norte-norte. Isso pode ser explicado pelas distâncias menores entre vários países do norte, mas também pela melhor infraestrutura de transportes e serviços. Além do mais, o desempenho logístico é encontrado como uma fonte significativa de variação dos custos comerciais sul-sul (ARVIS et al., 2012).

sofra do mesmo problema. Entretanto, a dificuldade de encontrar instrumentos válidos para os indicadores de competitividade impossibilitou o tratamento da endogeneidade neste trabalho. Por conta disso, os resultados aqui encontrados devem ser analisados levando em conta a possibilidade do viés. Wilson, Mann e Otsuki (2003) também indicam o problema da endogeneidade em suas estimativas. Para os autores é difícil encontrar, na prática, instrumentos que são exógenos às medidas de facilitação de comércio e aos fluxos de comércio. Assim, o uso de instrumentos não é uma solução eficaz para tratar a endogeneidade nesse caso. 
Quando um país desenvolvido importa de um país em desenvolvimento, o efeito do desempenho econômico do país importador aumenta o comércio. De modo oposto, se um país do sul importa de um país do norte, o comércio tende a reduzir com a melhoria do desempenho econômico do país do sul. Por isso, acredita-se que um melhor desempenho econômico nos países do sul aumenta seu consumo interno em detrimento das exportações.

Tabela 4 - Resultados estimados para a equação com os indicadores de competitividade

\begin{tabular}{|c|c|c|c|c|}
\hline \multirow{2}{*}{$\begin{array}{l}\text { Variável } \\
\text { Lnpibi }\end{array}$} & \multicolumn{2}{|c|}{ Efeitos fixos } & \multicolumn{2}{|c|}{ PPML } \\
\hline & $1,5894 * * *$ & $1,4150 * * *$ & $0,7474 * * *$ & $0,714201 * * *$ \\
\hline Lnpibj & $0,6163 * * *$ & $0,6200 * * *$ & $0,6456 * * *$ & $0,658624 * * *$ \\
\hline Lndistancia & $-1,2212 * * *$ & $-1,2490 * * *$ & $-0,8647 * * *$ & $-0,87468 * * *$ \\
\hline Lntarifa & $0,1183 * * *$ & $0,1346 * * *$ & $-0,0434 * * *$ & $-0,04319 * * *$ \\
\hline deco & $0,0064 * * *$ & $0,0075 * *$ & 0,0011 & 0,000344 \\
\hline egov & $-0,0333 * * *$ & $-0,0059$ & 0,0006 & $0,003937 * * *$ \\
\hline eneg & $0,0262 * * *$ & $0,0122 * * *$ & $0,0029 * *$ & 0,002356 \\
\hline infra & $-0,0261 * * *$ & $-0,0685 * * *$ & $-0,0021$ & $-0,00428 * * *$ \\
\hline nsdeco & . & $0,0114^{* * *}$ & . & $0,008733 * * *$ \\
\hline nsegov & . & $-0,0195 * * *$ & . & $-0,0039$ \\
\hline nseneg & . & 0,0053 & . & 0,003502 \\
\hline nsinfra & . & $0,0055^{*}$ & . & $-0,00503 * *$ \\
\hline sndeco & . & $-0,0135 * * *$ & . & $-0,00654 * *$ \\
\hline snegov & . & $-0,0381 * * *$ & . & $-0,00535^{*}$ \\
\hline sneneg & . & $0,0226 * * *$ & . & 0,000883 \\
\hline sninfra & . & $0,0675 * * *$ & . & $0,013016 * * *$ \\
\hline ssdeco & . & $-0,0061$ & . & 0,001951 \\
\hline ssegov & . & $-0,0418 * * *$ & . & $-0,00402$ \\
\hline sseneg & . & $0,0177 * * *$ & . & $-0,00113$ \\
\hline ssinfra & . & $0,0639 * * *$ & . & 0,003486 \\
\hline constante & $1,5894 * * *$ & $10,4446 * * *$ & $0,7474^{* * *}$ & $12,6588 * *$ \\
\hline $\mathrm{R}^{2}$ & 0,6221 & 0,6264 & 0,8884 & 0,9011 \\
\hline $\begin{array}{l}\text { Número de Ob- } \\
\text { servações }\end{array}$ & 51330 & 51330 & 51330 & 51330 \\
\hline
\end{tabular}

Fonte: Elaboração própria.

Nota: * $\mathrm{p}<0.10$; ** $\mathrm{p}<0.05 ;$ *** $\mathrm{p}<0.01$. Para o modelo de efeito aleatório é reportado o $\mathrm{R}^{2}$ "within". 


\section{Considerações Finais}

O aumento contínuo dos fluxos comerciais internacionais, somado aos esforços de liberalização, vivenciados nos últimos anos, destaca cada vez mais a importância de fatores que promovam a redução dos custos de comercialização. Uma parte significativa desses custos está relacionada à precária infraestrutura existente nos países, ao ambiente regulatório fraco, à ausência de transparência na aplicação de normas, à elevada burocracia e corrupção, entre outros, fatores que aumentam o preço das mercadorias e comprometem a competitividade dos países.

Atualmente, a política comercial não está ligada somente à tentativa de eliminação das barreiras comerciais. Os formuladores de políticas necessitam concentrar seus esforços também para melhorar o ambiente interno de negócios, o que, por sua vez, levaria à facilitação do comércio. Tendo em conta que as restrições diretas ao comércio na fronteira foram ou estão sendo eliminados por muitos países em desenvolvimento, torna-se evidente que a integração na economia mundial depende cada vez mais da eliminação das restrições por trás das fronteiras.

Os resultados encontrados neste estudo apontam que os indicadores de competitividade relativa desempenham um efeito positivo e significativo sobre os fluxos de comércio bilateral quando analisados de modo agregado. Os países têm muito a ganhar se direcionarem suas políticas para ações que promovam melhorias nos indicadores analisados. De modo geral, a relação positiva obtida entre os indicadores de competitividade e os fluxos de comércio sugere que os países podem ampliar o comércio através de reformas que gerem melhorias no ambiente doméstico e reduzam os entraves ao comércio internacional.

Reformas que visem melhorias internas das economias são capazes de elevar os fluxos de comércio bilateral, em um contexto em que as empresas realizem os seus negócios de forma inovadora, rentável e responsável. Aos governos fica a responsabilidade da proposição de políticas favoráveis à competitividade, a fim de que os recursos tecnológicos, científicos e humanos sejam usados de modo eficiente.

Cabe destacar que ainda há muito a se fazer nessa área. Fazem-se necessários estudos capazes de detalhar como os fatores relacionados ao ambiente interno dos países afetam o comércio bilateral, especificamente aqueles relacionados ao ambiente dos negócios e à eficiência dos governos. Certamente, dados primários, coletados nas diferentes indústrias, seriam muito importantes na análise da eficiência dos negócios, permitindo captar como as diferentes normas regulatórias afetam a competitividade.

Este trabalho sinaliza alguns dos possíveis impactos de melhorias nos diferentes fatores que compõem os indicadores de competitividade sobre os fluxos de comércio. A análise e classificação dos países conforme a competitividade pode ser 
vista como um meio de avaliar a potencialidade futura das economias e as oportunidades para o seu crescimento e desenvolvimento. Contudo, ainda há problemas metodológicos relacionados com a escolha dos indicadores, da sua importância relativa e da utilização de informações qualitativas. Desse modo, o desafio consiste em saber se o destaque na melhor colocação de um país é consistente com o desenvolvimento da economia mundial de longo prazo. Sugere-se que estudos futuros busquem métodos alternativos de quantificar essas medidas e, assim, possam contribuir para as discussões sobre os efeitos da competitividade doméstica sobre o comércio internacional.

\section{Referências}

ALBURO, F. A. Policy coherence and coordination for trade facilitation: integrated border management, single-windows and other options for developing countries. Bangkok: ESCAP, Aug. 2008. (Asia-Pacific Research and Training Network on Trade Working Paper Series, n. 57).

ANDERSON, J. E. A theoretical foundation for the gravity equation. The American Economic Review, v. 69, n. 1, p. 106-116, 1979. Disponível em: < http://www.jstor.org/stable/1802501>. Acesso em: 29 nov. 2011.

ANDERSON, J. E.; WINCOOP, E. Gravity with gravitas: a solution to the border puzzle. American Economic Review, v. 93, n. 1, p. 170-92, 2003. Disponível em: < http://fmwww. bc.edu/EC-P/WP485.pdf>. Acesso em: 29 nov. 2011.

Trade costs. Boston College Working Papers in Economics, Boston, n. 593, 2004. Disponível em: <http://ideas.repec.org/p/boc/bocoec/593.html > . Acesso em: 29 nov. 2011.

ARVIS, J. F. et al. Trade costs in the developing world: 1995-2010. Bangkok: ARTNeT, 2012. (Working Paper, n. 121). Disponível em: <www.artnetontrade.org>. Acesso em: 24 jan. 2013.

BALDWIN, R.; TAGLIONI, D. Gravity for dummies and dummies for gravity equations. Cambridge, MA: NBER, 2006. (NBER Working Paper, n. 12516), Disponível em: < http:// www.nber.org/papers/w12516>. Acesso em: 29 nov. 2011.

CENTRE D'ÉTUDES PROSPECTIVES ET D'INFORMATIONS INTERNATIONALES. Distances. 2011. Disponível em: < http://www.cepii.fr/anglaisgraph/bdd/distances.htm>. Acesso em: 01 out. 2011.

CHENG, I.; WALL, H. J. Controlling for heterogeneity in gravity models of trade and integration. Federal Reserve Bank of St. Louis Review, v. 87, n. 1, p. 49-63, 2005. Disponível em: <http://research.stlouisfed.org/publications/review/05/01/Cheng.pdf > . Acesso em: 29 nov. 2011.

CLARKE, G. R. G. Beyond tariff and quotas: why don't African manufacturing enterprises export more? Washington, D.C.: World Bank, 2005. (World Bank Policy Research, n. 3617),

DUVAL, Y. Trade facilitation beyond the Doha round of negotiations. In: UNITED NATIONS ECONOMIC AND SOCIAL COMMISSION FOR ASIA AND THE PACIFIC. Trade facilitation 
beyond the multilateral trade negotiations: regional practices, customs, valuation and other emerging issues. Bangkok: ESCAP, 2008. p. 1-25.

DUVAL, Y.; UTOKTHAM, C. Behind the border trade facilitation in Asia-pacific: cost of trade, credit information, contract enforcement and regulatory coherence. Bangkok: ESCAP, May, 2009. (Asia-Pacific Research and Training Network on Trade Working Paper Series, n. 67),

Beyond trade facilitation: impact of the domestic business environment on export competitiveness in Asia and the Pacific. In: MIKIC, M. (Ed.). Rising non-tariff protectionism and crisis recovery. Bangkok: ESCAP, 2010.

ENGMAN, M. The economic impact of trade facilitation. Paris: OECD, 2005. (OECD Trade Policy Working Papers, n. 21), Disponível em: <http://dx.doi.org/10.1787/861403066656>. Acesso em: 7 nov. 2011.

FEENSTRA, R. C. Advanced international trade: theory and evidence. Princeton, NJ: Princeton University Press, 2004.

FRANÇOIS, J.; MANCHIN, M. Institutional quality, infrastructure and the propensity to export. London: CEPR, 2006. (CEPR Discussion Paper, n. 6068),

GREENE, W. H. Econometric analysis. 5. ed. Upper Saddle River: Prentice-Hall, 2003.

GUJARATI, D. N. Econometria Básica. 5. ed. Porto Alegre: AMGH, 2011.

HAMMAR, T. Trade facilitation in Vietnam: recent progress and impact. Lund: Department of Economics at the University of Lund, 2009. (Minor Field Study Series, n. 190).

HOEKMAN, B.; NICITA, A. Trade policy, trade costs and developing country trade. Washington, D.C.: World Bank, 2008. (World Bank Policy Research Working Paper, n. 4797),

INTERNATIONAL INSTITUTE OF MANAGEMENT AND DEVELOPMENT. IMD World Competitiveness Yearbook 2011. Lausanne: IMD, 2011.

. IMD World Competitiveness Online 1995-2011. 2011. Disponível em: <https://www. worldcompetitiveness.com>. Acesso em: 01 out. 2011.

IWANOW, T.; KIRKPATRICK, C. Trade facilitation, regulatory quality and export performance. Manchester: Impact Assessment Research Centre, 2007. (Working Paper Series, n. 19). Disponível em: <http://www.sed.manchester.ac.uk/research/iarc/pdfs/TI_CK_TF_ Regulation.pdf>. Acesso: 11 set. 2012.

LIMÃO, N.; VENABLES, A. J. Infrastructure, geographical disadvantage, transport costs and trade. The World Bank Economic Review, v. 15, n. 3, p. 451-479, 2001. Diponível em: < http:// economics.ouls.ox.ac.uk/12078/1/INFRAS.pdf>. Acesso: 11 set. 2012.

PAULA, J. S. Indicadores internos de competitividade e seus efeitos nos fluxos de comércio. 2013. Dissertação (Mestrado em Economia) - Departamento de Economia, Universidade Federal de Viçosa, Viçosa, MG, 2013. 
SANTOS SILVA, J. M. C.; TENREYRO, S. The log of gravity. The Review of Economics and Statistics, v. 88, n. 4, p. 641-658, 2006. Disponível em: <http://www.mitpressjournals.org/doi/ abs/10.1162/rest.88.4.641 >. Acesso em: 6 jul. 2012.

SHEPHERD, B.; WILSON, J. S. Trade facilitation in ASEAN member countries: measuring progress and assessing priorities. Washington, D.C.: World Bank, 2008. (Policy Research Working Paper Series, n. 4615),

SOUZA, M. J. P. Impactos da facilitação de comércio sobre os fluxos de comércio internacional: evidências do modelo gravitacional. 2009. Tese (Doutorado em Economia Aplicada) - Escola Superior de Agricultura Luiz de Queiroz, Universidade de São Paulo, Piracicaba, SP, 2009.

SOUZA, M. J. P.; BURNQUIST, H. L. Facilitação de comércio e impactos sobre o comércio bilateral. Estudos Econômicos, v. 41, n. 1, p. 91-118, 2011.

STAPLES, B. R. Trade facilitation: improving the invisible infrastructure. In: HOEKMAN, B. et al. (Ed.). Development, trade and the WTO: a handbook. Washington, D. C.: World Bank, 2002.

WILSON, J. S., MANN, C. L.; OTSUKI, T. Assessing the potential benefit of trade facilitation: a global perspective. Washington, D.C.: World Bank, 2004. (World Bank Policy Working Paper, n. 3224).

. Trade facilitation and economic development -measuring the impact. Washington, D.C.: World Bank, 2003. (Policy Research Working Paper, n. 2988).

WORLD INTEGRATED TRADE SOLUTION DATABASE. 2011. Disponível em: < http://wits. worldbank.org/wits>. Acesso em: 01/10/2011.

WORLD TRADE ORGANIZATION. Trade facilitation. 2011. Disponível em: < http://www. wto.org/english/tratop_e/tradfa_e/tradfa_intro_e.htm >. Acesso em: 1 out. 2011.

Recebido em: 24/04/2013. Aceito em: 07/10/2014. 